
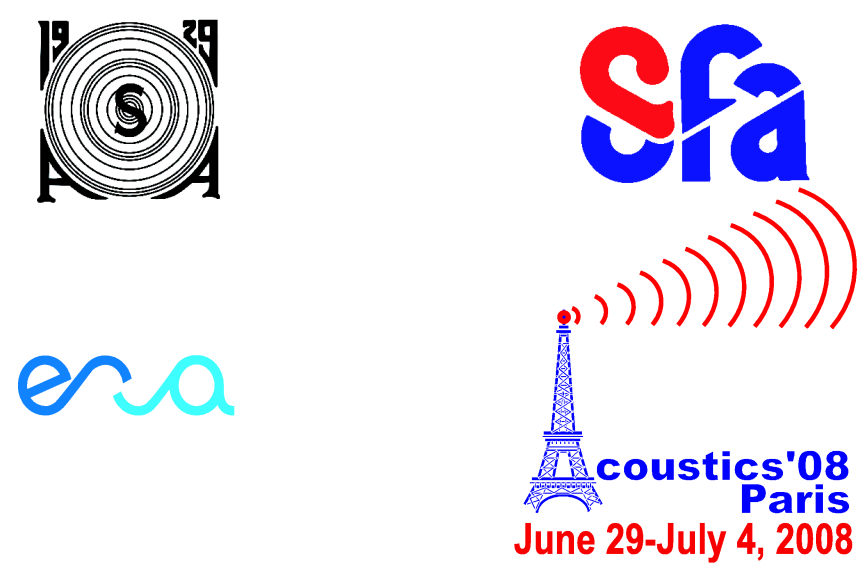

EAA

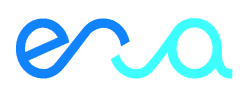

June 29-July 4, 2008

www.acoustics08-paris.org

\title{
euronoise
}

\section{Using the MBES for classification of riverbed sediments}

\author{
A. Amiri-Simkooei ${ }^{\mathrm{a}}$, M. Snellen ${ }^{\mathrm{a}}$ and D. G Simons ${ }^{\mathrm{b}}$
}

${ }^{a}$ Acoustic Remote Sensing Group, Delft Institute of Earth Observation and Space Systems, Delft University of Technology, Kluyverweg 1, 2629 HS Delft, Netherlands

${ }^{b}$ Delft University of Technology, P.O. Box Postbus 5048, 2600 GA Delft, Netherlands a.amirisimkooei@tudelft.nl 
For keeping the Dutch rivers suitable for commercial activities bottom stabilizing measures are required. For example, the bottom of the river Waal, connecting Rotterdam with German industrial areas, is subsiding. Since the subsidence varies along the river, dangerous shoals occur. Sediment suppletions are planned to counteract the subsidence. Appropriate suppletion material is expected to keep the bottom more stable. To monitor the suppletion effectiveness, multibeam echosounder (MBES) measurements are planned, allowing for simultaneous estimation of bathymetry and sediment composition. For the latter, we apply a method employing the MBES backscatter data. It estimates the number of sediment types present in the survey area and discriminates between them by applying the Bayes decision rule for multiple hypotheses, implicitly accounting for the backscatter strength ping-to-ping variability. The method's applicability was demonstrated in a well-surveyed test area (North Sea). In 2007, MBES measurements were acquired at the Waal, accompanied with extensive sediment grabbing. Contrary to the test area, water depths are very shallow and significant bottom slopes exist, requiring corrections. The lower water depths correspond to smaller beam-footprints, resulting in a higher ping-to-ping variability. Consequently the discriminating power between sediments will decrease. The performance of the classification method for this river environment is assessed.

\section{Introduction}

It is widely accepted that multi-beam echo sounder (MBES) data can be used to measure the bathymetry, and to study statistical characteristics of acoustical signals backscattered from the seafloor. Data acquired from MBES are employed to obtain information about the physical properties of the riverbed and seafloor. The main advantage of the method is its high coverage capabilities with limited costs.

The backscatter intensity varies with incidence angle. To eliminate this angular dependence, one can for instance apply the Lambert's law. The angular dependence of the backscatter data can also potentially be used as a tool for classification. A problem in this approach arises for areas where the seafloor type varies along the swathe. It is therefore difficult to discriminate between the angular variation and the real seafloor type variation along the swathe. In an earlier work, a method was proposed for the classification of the seabed sediment (see [1]). The method, based on the Bayesian decision rule, was applied to MBES backscatter data for the classification in a well-known test area in the North Sea. This method employs the backscatter data per angle instead of using the angular behavior of the backscatter strength. It accounts for statistical fluctuations in backscatter strength, i.e. ping-to-ping variability for fixed seafloor type.

There are some issues when dealing with riverbed classification in very shallow waters, which we need to come up with. The classical Rayleigh distribution is not applicable to backscattered data when the deterministic number of scatterers within the resolution cell (also called ensonified area, signal footprint, or size of scatter pixel) is not large enough and hence the central limit theorem does not hold.

MBES measurements acquired at the river Waal in the Netherlands in 2007, also accompanied with extensive sediment grabbing, are carried out at very shallow water and significant bottom slopes exist. The lower water depths correspond to smaller beam footprints, resulting in a higher ping-to-ping variability. Consequently the discriminating power between sediments will decrease. The performance of the classification method for this river environment is assessed.

The objective of this contribution is twofold. First, we briefly describe the MBES classification method, and apply it to the data set from the river Waal. For this area, extensive sediment grabbing is also available, which allows for an assessment of the classification method performance. Then, we explain the problem with another class of distributions, namely nonRayleigh distributions. The possible application of the K-distribution for the classification of the Waal data is assessed.

\section{Statistical characteristics of backscatter data}

The echo amplitudes measured by the MBES are employed for seafloor and riverbed classification. The MBES systems that typically operate at a few $100 \mathrm{kHz}$ permit seafloor backscatter imaging with a high resolution. Since the ensonified area $A$ (i.e. the signal footprint) is small compared with the beam footprint for beams away from nadir, many scatter pixels are expected to fall within the footprint of the receiving beam. This does not, however, hold for the very shallow water as we deal with in the river Waal. The number of scatter pixels $N$ is not large enough in order to use the central limit theorem, and hence the non-Rayleigh distributions should be used.

The data employed for the classification method consist of backscatter values (in dB) per receiver beam, i.e. backscatter values obtained from averaging over $N$ independent scatter pixels. Such values - given for each beam angle - are corrected for propagation loss and the ensonified area $A$. The averaged backscatter value is subject to statistical fluctuations and there is a pingto-ping variability masking the influence of the seafloor type on backscatter intensity.

It is traditionally assumed that the backscatter intensity of the $i^{\text {th }}$ scatter pixel in a beam, denoted by the random variable $I$ is exponentially distributed [2] (or Chi-square distribution with 2 degrees of freedom). This is based on the validity of the central limit theorem where the number of scatterers $N_{s}$ inside the resolution cell is large enough. The normalized amplitude $\sqrt{I}$ has then a Rayleigh distribution. The corresponding backscatter strength in $\mathrm{dB}$ is given as: $B S=\log _{10}(I)$ and has a Gumbel distribution, which is a peaked asym- 
metric distribution. The Gumbel distribution is a special case of the log-Weibull distribution.

\subsection{Classification methodology}

When the number of scatter pixels $N$ is large enough, based on the central limit theorem, the averaged backscatter strength $\overline{B S}$ (per beam footprint) has a Gaussian distribution. The classification approach proposed by [1] employs the averaged backscatter data at a single angle. Without going into the details, the method comprises the following steps:

Step 1 (nonlinear curve fitting): The algorithm starts by fitting $r$ number of Gaussian PDF, i.e. $\sum_{i=1}^{r} c_{i} \mathrm{~N}\left(\mu_{i}, \sigma_{i}^{2}\right)$, to the histogram of selected measured backscatter strengths, where each PDF represents one seafloor type. For each Gaussian distribution, the mean and the variance are unknown. The linear combination of the PDFs is also not known. This leads to the total number of unknown parameters as $n=3 r$ (i.e. $c_{i}$, the contribution of individual PDF, $\mu_{i}$, the mean of PDF, and $\sigma_{i}$, the standard deviation of PDF, $i=1, \ldots, r)$.

Step 2 (classes identification): For the classification, when we know the PDF for each seafloor type $i$, we can apply the Bayes decision rule. We have $r$ hypotheses $\mathrm{H}_{i}$, $i=1, \ldots, r$, and therefore there exist $r$ possible decisions. We choose the hypothesis that, given the observation $y$, maximizes the likelihood function. The intersections of the $r$ Gaussian PDFs result in $r$ non-overlapping acceptance regions.

Step 3 (assigning seafloor types): We need to assign a seafloor type to each of the $r$ acceptance regions obtained in the previous step.

Step 4 (quality assessment): The quality of the classification algorithm can be assessed by calculating the decision matrix of the multiple-hypothesis-testing problem. This matrix contains the probabilities of correct and incorrect decision. The decision matrix provides a measure of the quality of the classification algorithm and can be calculated prior to the actual mapping part of the algorithm. If the probability of incorrect decision decreases, the power of the discrimination will increase.

Step 5 (mapping): This final step of the algorithm comprises the actual mapping, i.e. allocation of seafloor type (e.g. a colour) to all backscatter strength data points. As the MBES system provides a position to each backscatter strength measurement, we can map seafloor type versus position.

\subsection{K-distributed backscatter intensity}

The classical Rayleigh distribution theory is not applicable, at least, when

- the seafloor and hence seafloor data are rough,

- the number of scatterers $N_{s}$ is a random variable with high variance,

- the number of scatterers within the resolution cell is not large enough,

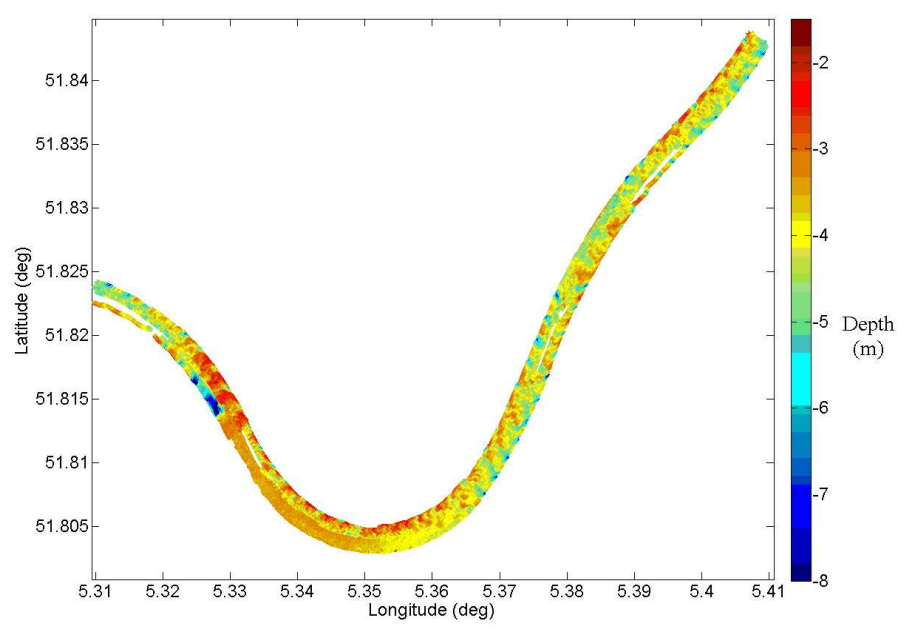

Figure 1: Bathymetry map of river Waal (in meter).

- the assumption of "independent and identically distributed' is violated,

Statistical analysis of backscatter intensity typically deals with fitting a set of theoretical distributions, to see which one describes data the best. Non-Rayleigh distributions (e.g. K-distribution) can better fit the skewed distributions and provide new parameters for characterization. It is shown that the K-distribution parameters depend on the incident angle.

It is widely accepted to use the K-distribution when the classical Rayleigh distribution is not applicable to backscatter amplitudes. The K-distribution is ([3])

$$
f_{I}(I)=\frac{2\left(\frac{N \nu}{\mu}\right)^{\frac{N+\nu}{2}} I^{\frac{N+\nu-2}{2}}}{\Gamma(\nu) \Gamma(N)} \mathrm{K}_{\nu-N}\left(2 \sqrt{\frac{N \nu}{\mu} I}\right)
$$

where $\mu$ is the scale parameter, $\nu$ is the shape parameter, $N$ is the multilook parameter (i.e. the number of scatter pixels in the beam footprint), and $\mathrm{K}_{\nu-N}$ is the modified Bessel function of the second kind. This Kdistribution results from two independent $\Gamma$-distributed random variables. The K-distribution has proved to be a promising and useful model for backscattering statistics in MBES and side-scan sonar data $[4,5]$.

The maximum likelihood estimation method is usually applied to estimate the parameters $(\mu$ and $\nu)$ of the K-distribution [6]. An alternative is based on the method of moments. We use a method which is based on the least-squares principle; fitting a curve to the histogram of the data in a least-squares sense. Such estimates are first of all independent of the distribution of the data, second they are unbiased, and third they give the best possible precision for the unknown parameters.

\section{Results on MBES data}

\subsection{Experiment description}

The river Waal is the main distributary branch of river Rhine flowing to the central Netherlands for about 80 $\mathrm{km}$. It is a major river that serves as the main waterway connecting the Rotterdam harbor and Germany. 

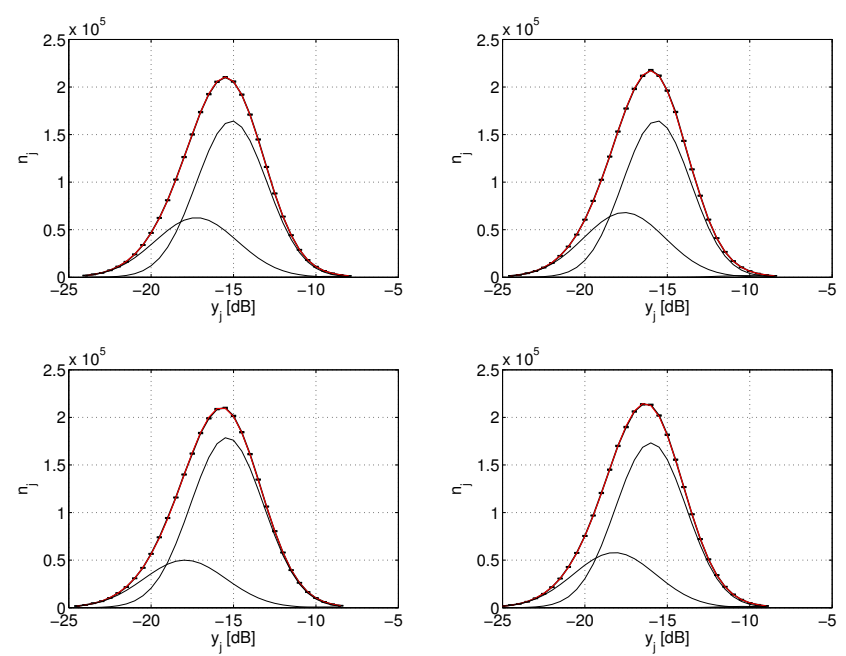

Figure 2: Histogram of measured backscatter data at $\theta=62^{\circ}$ (top) and $\theta=60^{\circ}$ (bottom) over the whole area; left and right transducers; number of Gaussians $r=3$.
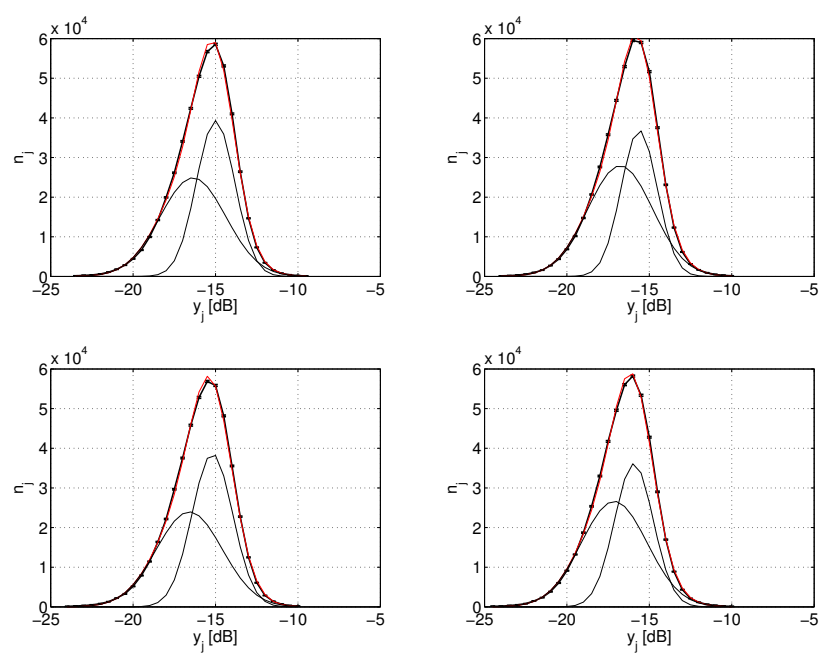

Figure 3: Histogram of averaged backscatter data (over a small window) at $\theta=62^{\circ}$ (top) and $\theta=60^{\circ}$ (bottom) over the whole area; number of Gaussians $r=2$.

The bottom of the river is subsiding. Since the subsidence varies along the river, dangerous shoals can occur. Sediment suppletions are planned to counteract the subsidence. Appropriate suppletion material is expected to keep the bottom more stable. To monitor the suppletion effectiveness, multibeam echosounder (MBES) measurements are executed, allowing for simultaneous estimation of bathymetry and sediment composition.

In October 2007, MBES measurements were acquired at the Waal, accompanied with extensive sediment grabbing. The MBES used for the measurements is an EM3002, typically working at a frequency of 300 $\mathrm{kHz}$ for shallow water; the pulse length is $150 \mu \mathrm{s}$; the maximum number of beams per ping is 254; and the maximum ping rate is $40 \mathrm{~Hz}$. Figure 1 shows the resulting bathymetry. This contribution presents some preliminary results on the classification, and provides some outlook for future research.
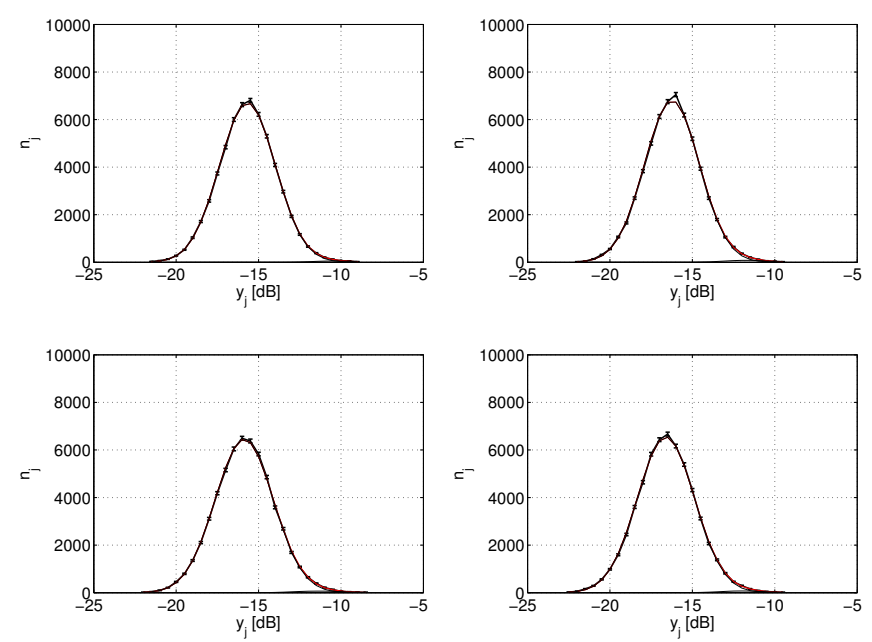

Figure 4: Histogram of averaged backscatter data at $\theta=62^{\circ}$ (top) and $\theta=60^{\circ}$ (bottom) over a flat area; number of Gaussians $r=2$.
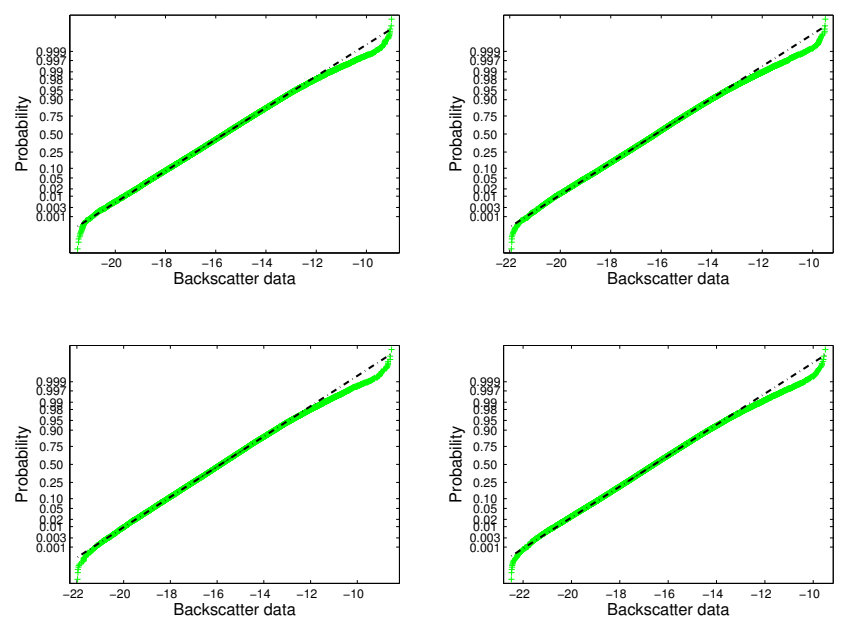

Figure 5: Normal probability plot of averaged backscatter data at $\theta=62^{\circ}$ (top) and $\theta=60^{\circ}$ (bottom) over a flat area; number of Gaussians $r=1$.

\subsection{Classification results}

We now apply the classification method of the previous section into the measured backscatter data. Figure 2 shows the histogram along with its best Gaussians fit for the backscatter values at $\theta=60^{\circ}$ and $\theta=62^{\circ}$. The results indicate that there exist 3 sediment types for the riverbed (3 Gaussians found; the third PDF is very small). One can do the classification based on these results.

There are however a few issues that need to be addressed here. Water depths are very shallow and significant bottom slopes exist. The low depth results in a small number of scatterers within the resolution cell $\left(N_{s}\right.$ is low), and a small number of scatter pixels per beam footprint ( $N$ is low). In both cases, the central limit theorem is not valid, and neither is the normality assumption of the backscatter data. These will affect the classification results. On the other hand, the lower wa- 


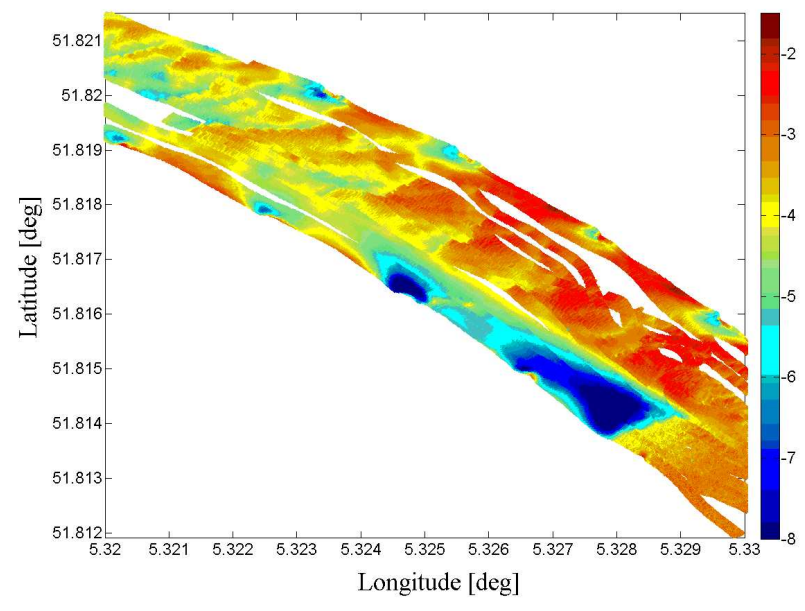

Figure 6: Bathymetry of the river Waal; typical area longitude from 5.32 to $5.33^{\circ}$ (unit in meter).

ter depths correspond to smaller beam footprints. This results in a higher ping-to-ping variability (higher variance), and therefore the discriminating power will decrease.

One way out of these dilemmas is to increase the number of samples for each beam considered. This is achieved by including more angles around the central beam angle (e.g. with deviation of $1^{\circ}$ ). For such close angles, usually, the angular dependence of the backscatter distribution is negligible. One can also average over the close pings as the ping rate is high $(40 \mathrm{~Hz})$. These result in a window that contains, say, 30 samples. By doing this we are able to

- restore the normality assumption by means of the central limit theorem, and

- decrease the variance and hence increase the discriminating power between sediments.

Figure 3 shows the histogram and its best Gaussians fit for the backscatter values after applying this idea. The histograms are more peaked than those in Fig. 2, indicating lower variance. To test the normality assumption of the averaged data we considered the southern part of a smaller area where the longitude lies between 5.33 to $5.36^{\circ}$ - a stable flat area. The corresponding histogram along with two Gaussian PDFs is shown in Fig. 4. The second PDF is really small and likely describes the heterogeneity of the sediment type. This can also be illustrated using the 'normal probability plot' for the backscatter data (Fig 5). Small deviations are observed at the right hand sides of the plots.

We now apply the classification method to the averaged backscatter data on a small area where the longitude lies between 5.32 and $5.33^{\circ}$. The bathymetry is given in Fig. 6. The results of the classification method are given in Fig. 7. Two sediment types are identified by colors (red dots represent high values, i.e. $\mathrm{BS} \approx-15 \mathrm{~dB}$; green dots represent low values, i.e. $\mathrm{BS} \approx-18 \mathrm{~dB}$ ). $\mathrm{A}$ significant correlation between the bathymetry and the classification results is observed; the deeper the depth is, the larger the backscatter values will be.

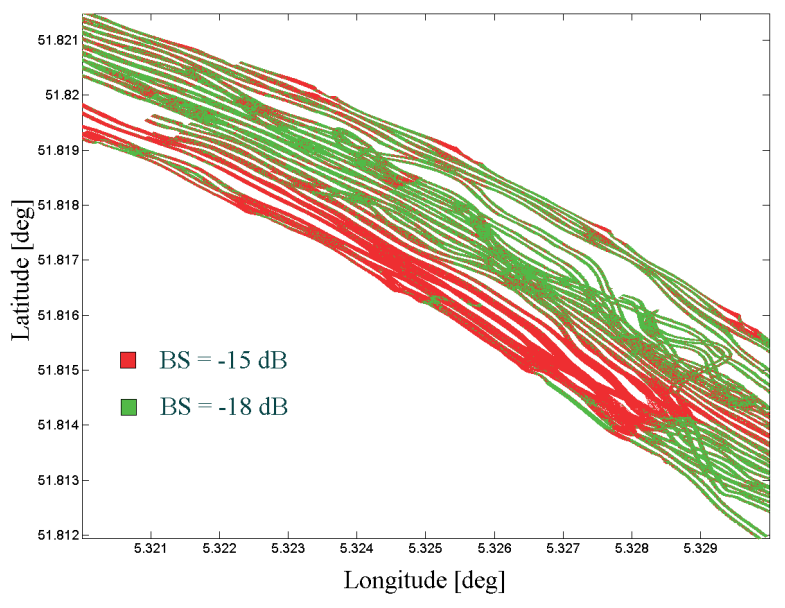

Figure 7: Classification map obtained from backscatter data obtained at $\theta=62,60,58,56,54,51,48,45,42,39$, and $36^{\circ}$. For each angle separate classification has been applied and results put in a single figure.

\subsection{Distribution issues}

Another class for the distribution of the backscatter data is now considered. It is the K-distribution, which holds for backscatter intensities when the classical Rayleigh distribution is not valid. The K-distribution is compared to the experimental PDFs. Figure 9 shows typical graphical examples of the observed backscatter (amplitude) along with their least-squares fit. The fit is not good enough (large $\chi^{2}$ value), which is a further indication for having more than one sediment types (this came out of the Gaussian fitting).

The angular evolution of backscattering statistics via the evolution of one parameter (the shape parameter $\nu$ in Eq. 1) of the K-distribution is investigated. Its estimate is based on the least-squares curve fitting using the simultaneous estimation of the shape parameter $\nu$ and the scale parameter $\mu$. The results, for $\nu$, are shown in Fig. 8. The angular evolution of the shape parameter $\nu$ coincides with the findings of [5] and [4]. For the intermediate incidence angles, the shape-parameter values are low. At grazing angles, the increase is due to the extension of the resolution cell, which includes a greater number $N_{s}$ of scatterers; the central limit theorem applies, and the K-distribution tends to an exponential distribution (and correspondingly the Rayleigh distribution for amplitude).

The results given in [4] show a point where the functional behavior of the shape-parameter curves (i.e. $\nu=\nu(\theta))$ reverse for soft sediments, and the rough seafloors do not seem to exhibit this transition angle. This indicates that our seafloor can be considered as a rough seafloor (we cannot see such transition point here as the shape parameter increases with $\theta$ ). We also observe that the shape parameters, in the stable flat area, are significantly smaller than their corresponding values in the entire area (Fig. 8). This can be considered as the effect of the significant bottom slopes, which have not been considered in this study. 


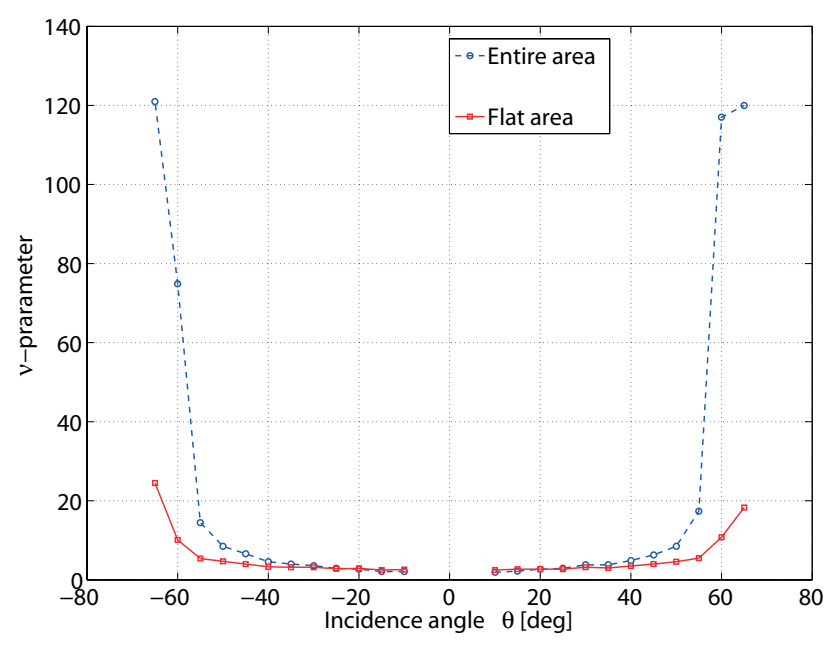

Figure 8: Angular evolution of shape parameter $\nu$ of $\mathrm{K}$-distribution for entire area and flat area.

\section{Concluding remarks}

To monitor Dutch rivers, multibeam echosounder (MBES) measurements allow one to simultaneously estimate bathymetry and sediment composition. The bathymetry results show that the sediment suppletions planned to counteract the subsidence in the river Waal are still stable (we observed a stable flat area, longitude from 5.33 to $5.36^{\circ}$ in Fig. 1).

Riverbed classification using the MBES backscatter data is considered to be a promising approach. This contribution highlighted a few problems that one usually deals for shallow water classification, as we deal, for instance, with MBES measurements acquired at the Waal. The classification method proposed by [1] was applied to the Waal data.

There are still issues that we need to come up with. We should note that the degree to which different seafloor types can be discriminated using the statistical characteristics of the backscatter data depends on

- geoacoustical features of the seafloor types. An obvious effect is on the mean and variance of the backscatter data,

- degree of the roughness of the seafloor, number of scatterers $N_{s}$ in the resolution cell, etc. These usually affect the distribution itself (e.g. exponential distribution versus K-distribution), and

- presence of local slopes of the seafloor. In principle, the backscatter data should be corrected for the bottom slopes. However, the differential slopes will significantly affect the distribution parameters.

As a next step, we consider correcting the backscatter data for the significant local slopes, and then apply the classification method.

Finally, we will also compare the classification results with the available sediment grabbing.
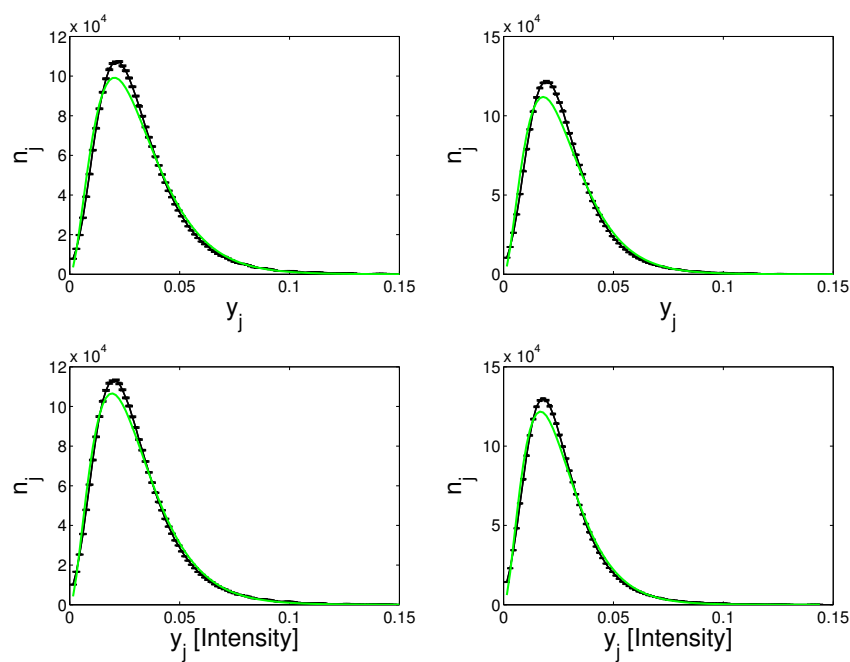

Figure 9: Measured histogram of backscatter intensity and statistical K-distribution fitting; $\theta=62^{\circ}$ (top), $\theta=$ $60^{\circ}$ (bottom).

\section{Acknowledgments}

The financial support comes from the Dutch Ministry of Transportation and Water Management (Rijkswaterstaat). We would like to acknowledge Dr. Arjan Sieben, Ir. Ben Dierikx, and Adri Wagener for providing the Waal data.

\section{References}

[1] D. G. Simons and M. Snellen. A Bayesian approach to seafloor classification using multi-beam echo-sounder backscatter data. Applied Acoustics, Under review, 2008.

[2] F. T. Ulaby, T. F. Haddock, and R. T. Austin. Fluctuation statistics of millimeter-wave scattering from distributed targets. IEEE transactions on Geoscience and Remote Sensing, 26(3):268-281, 1988.

[3] E. Jakeman. Non-Gaussian models for the statistics of the scattered waves. Advances in Physics, 37(5):471-529, 1988.

[4] G. Le Chenadec and J. M. Boucher. Angular dependence of $K$-distributed sonar data. IEEE Transactions on Geoscience and Remote Sensing, 45(5), 2007.

[5] L. Hellequin and J. M. Boucher. Processing of highfrequency multibeam echo sounder data for seafloor characterization. IEEE Journal of Oceanic Engineering, 28(1):78-89, 2003.

[6] I. R. Joughin, D. B. Percival, and D. P. Winebrenner. Maximum likelihood estimation of $\mathrm{K}$ distribution parameters for SAR data. IEEE transactions on Geoscience and Remote Sensing, 31(5):989-999, 1993. 